\title{
Materiales para la educación sexual en espacios no formales: el Conseyu de la Mocedá d'Asturies
}

\author{
Diego Fernández Fernández, María Rodríguez Suárez e Iván Gómez Beltrán*
}

\author{
PALABRAS CLAVE \\ Educación sexual mediática \\ Conseyu de la Mocedá \\ Materiales educativos \\ Educación no formal \\ Formación continua
}

\section{KEYWORDS}

Media sex education

Conseyu de la Mocedá

Educational materials

Non-formal education

Lifelong education

\begin{abstract}
RESUMEN
El objetivo de este estudio es poner en valor el papel de los espacios educativos no formales en la elaboración de materiales relativos a la educación sexual mediática. La metodología planteada se basa en un estudio de caso centrado en el Consejo de la Juventud del Principado de Asturias y, en concreto, en materiales destinados al profesorado y personal educador elaborados entre el año 2014 hasta la actualidad. En los resultados se analizan cinco guías que prestan atención a los elementos mediáticos que consumen las personas jóvenes y que puedan adaptarse mejor al desarrollo, las vivencias y las problemáticas derivadas de la convivencia cotidiana con las TRICs (Tecnologías de la Relación, la Información y la Comunicación). Se concluye que los materiales planteados se presentan como recursos útiles para abordar la educación sexual por su facilidad de acceso y por un diseño teórico que garantiza abordar de manera directa y cercana las necesidades tanto del profesorado como del alumnado.
\end{abstract}

\section{Materials for sex education in non-formal education: the Asturian Youth Council}

\section{ABSTRACT}

The aim of this study is to highlight the role of non-formal educational spaces in the development of materials related to media sex education. The proposed methodology is based on a case study centred on the Youth Council of the Principality of Asturias and, specifically, on materials aimed at teachers and educators produced between 2014 and the present. The results analyse five guides that pay attention to the media elements that young people consume and that can be better adapted to the development, experiences and problems derived from the daily coexistence with TRICs. It is concluded that the materials presented are presented as useful resources to address sexuality education due to their ease of access and a theoretical design that guarantees a direct and close approach to the needs of both teachers and students.

Conseyu de la Mocedá del Principáu d'Asturies

Autor de correspondencia: * Iván Gómez Beltrán; ivangom1@hotmail.com

Recibido: 27/05/2021 - Aceptado: 01/09/2021

Revista de Formación del Profesorado e Investigación Educativa

Facultad de Formación del Profesorado y Educación

Universidad de Oviedo

Enero - Diciembre 2021

ISSN: 2340-4728

(c) (1) () $\odot$ Esta obra está bajo una licencia internacional Creative Commons

Esta obra está bajo una licencia internacional
Atribución-NoComercial-SinDerivadas 4.0. 
Introducción: La importancia de la educación sexual en la actualidad

En los últimos años, asociaciones internacionales como la UNESCO (2016) han reconocido los considerables avances que los países europeos han efectuado respecto a la implementación de la educación sexual, al igual que en el desarrollo de medidas para integrar dicha materia en los currículos escolares y en la formación del profesorado. Sin embargo, algunas investigaciones como las elaboradas por Soares y Toledo (2012), Bejarano y Mateos (2016), Fernández y Calvo (2020) y Calvo (2021) apuntan a que la situación de la educación sexual en España no ha sufrido modificaciones sustanciales y que los escasos progresos normativos en materia de educación sexual no responden a las nuevas realidades donde la diversidad de los sexos y las sexualidades es cada vez más evidente.

Para garantizar el bienestar y la vivencia positiva de la sexualidad existe un consenso internacional que se materializa en los Derechos Sexuales y Reproductivos, comprendidos en el marco de los Derechos Humanos. Conocer y comprender la importancia de los Derechos Sexuales y Reproductivos es clave para disfrutarlos, pero también para exigirlos, para denunciar cuando se produzcan situaciones de vulneración o cuando no se realizan las acciones concretas necesarias para su disfrute. Por ello, se considera necesario generar y transmitir en contextos formales, no formales e informales las evidencias y el conocimiento existente en torno a la educación sexual inclusiva como estrategia fundamental para garantizar el pleno desarrollo y disfrute de los Derechos Sexuales y Reproductivos.

Existen numerosos documentos y proyectos que cuentan con el apoyo de entidades internacionales de prestigio, como la UNESCO, la OMS o la SEDRA-Federación de Planificación Familiar -entre otras-, y cuya meta común y coordinada es la progresiva implantación de la educación sexual en los sistemas educativos del mundo, incluyendo el español. No obstante, "hoy en día sigue sin asumirse de manera global por una ciudadanía que la mira desde supuestos más morales e ideológicos que académicos" (Calvo, 2021: 283). Esta situación evidencia que todavía queda mucho camino por recorrer para que la educación sexual sea un derecho accesible para todas las personas. Concretamente, en el sistema educativo español se encuentra en una clara incongruencia legislativa (Lameiras et al., 2016), lo que significa que las demandas y avances sociales no van acompañados de cambios legales efectivos (Bejarano y Jiménez, 2016; Fernández, 2018).

\section{La legislación en materia de educación sexual}

Como evidencian los estudios de Soares y Toledo (2012), Bejarano y Mateos (2016), Fernández y Calvo (2020) y Calvo (2021), la educación sexual no tiene cabida en el currículum de la educación pública en España, quedando relegada a un abordaje meramente anecdótico. La Ley Orgánica de Mejora de la Calidad Educativa (LOMCE), aprobada en 2013, que hasta 2020 estructuraba el actual sistema educativo, parcela sus contenidos en piezas informativas recogidas en la materia de Biología, en la etapa de Secundaria (Bejarano y García, 2016; Bejarano y Jiménez, 2016; Lameiras et al., 2016; Calvo, 2021), a pesar incluso de que la propia norma aboga por la "formación permanente del profesorado" (Ley Orgánica de La Mejora de la Calidad Educativa, 2013, Artículo 111 bis).

La nueva Ley Orgánica de Modificación de la LOE (LOMLOE), aprobada en 2020 y que derogó la LOMCE, obliga en numerosos artículos al abordaje explícito dentro y fuera del currículum educativo de contenidos, valores y actitudes relacionados con la sexualidad. No obstante, el abordaje de la educación sexual se hará, por lo que refleja el texto legal, como un contenido concreto dentro de la materia "Educación para la Salud" y, en todo caso, de forma transversal. Otra norma que incluye dentro del sistema educativo la formación en salud sexual y reproductiva es la Ley
Orgánica de Salud Sexual y Reproductiva y de la Interrupción voluntaria del Embarazo de 2010 (Garzón, 2016). Este marco legal contempla la educación sexual como la estrategia pedadógica más valiosa para abordar las distintas facetas de la salud sexual y reproductiva y de las problemáticas contemporáneas, especialmente en torno a los embarazos no planificados y a las infecciones de transmisión sexual. Se establece un objetivo fundamental para que los poderes públicos lleven a cabo diferentes actuaciones para promover, entre otras, el acceso universal a servicios y programas de esta índole vinculados con la salud sexual y reproductiva y desde la perspectiva de género.

Tras este breve recorrido, podemos observar que la propuesta legal española no garantiza la inclusión de la educación sexual dentro del currículum escolar, siendo esta limitada e insuficiente y dejándola en manos de la voluntariedad del profesorado (Martínez et al., 2011). La educación sexual todavía se encuentra en proceso de ser trabajada en el sistema educativo español de forma científica, rigurosa, sistemática, accesible y desde un modelo biográfico-profesional comprensivo y con perspectiva de género (Lameiras et al., 2016).

\section{El Conseyu de la Mocedá del Principáu d'Asturies y el Grupo de Salud}

A pesar de transitar por un panorama donde la educación sexual no es un derecho accesible para todas las personas, Calvo afirma que "existe un amplio número de agentes, instituciones públicas para la salud, la educación y el desarrollo; organizaciones no gubernamentales centradas en el trabajo con menores y jóvenes, asociaciones y agrupaciones socioculturales de todo tipo" que tienen como objetivo suplir esta carencia (2021: 282). Es aquí donde podemos enmarcar el Conseyu de la Mocedá del Principáu d'Asturies (CMPA), un organismo autónomo del Principado de Asturias adscrito a la Consejería de Presidencia, con personalidad jurídica propia y plena capacidad para el cumplimiento de sus fines. Se regula por la Ley del Principado de Asturias 6/2019, de 29 de marzo, de Participación y Promoción Juvenil y su principal objetivo es ejercer de interlocutor para que las personas jóvenes de Asturias sean escuchadas por la Administración Autonómica y la opinión pública en todo aquello que les afecte, promoviendo actividades dirigidas a asegurar la participación, la información y la formación juvenil.

A nivel estatal, se integra dentro del Consejo de la Juventud de España (CJE), una plataforma de entidades juveniles creada en 1983 y formada por los Consejos de Juventud de las Comunidades Autónomas y organizaciones juveniles de ámbito estatal, entre cuyos objetivos se encuentran colaborar con los agentes sociales proporcionando instrumentos para conseguir una política juvenil global que dé respuesta a los problemas, demandas e inquietudes de las personas jóvenes fomentando la participación juvenil (Consejo de la Juventud de España [CJE], s.f.).

Dentro de la estructura organizativa del CMPA se encuentran los grupos de trabajo, entre los que destaca el Grupo de Salud que pretende promover hábitos de vida saludable y que es el encargado de ofrecer herramientas y recursos relacionados con la educación y la salud sexual. Las funciones principales de este órgano de trabajo son dos: por un lado, potenciar la participación transformadora de la juventud en la sociedad. Esto se consigue a través de la intervención de mediadoras y mediadores juveniles que trabajan directamente con personas jóvenes en centros educativos, escuelas-taller o el tejido asociativo, en el marco del Plan Sexual de la Consejería de Salud y Servicios Sanitarios. Por otro lado, este área también funciona como un centro de recursos y formación continua para asociaciones juveniles, profesionales de la educación y de la salud y otros agentes sociales que trabajen con población joven (Conseyu de la Mocedá del Principáu d'Asturies [CMPA], s.f.). Los materiales a los que nos referimos en este análisis se enmarcan en este contexto: son instrumentos para la formación de formadores. 


\section{Objetivos}

El objetivo principal de este estudio es realizar un análisis descriptivo de los principales materiales elaborados por el Conseyu de la Mocedá en lo referente a la formación de formadores en educación sexual mediática en Asturias. Entre los objetivos específicos, se encuentran:

- Poner en valor la producción de materiales elaborados desde espacios educativos no formales que sirvan como complemento formativo del currículum académico.

- Evidenciar la cercanía de los espacios educativos no formales con la realidad educativa del profesorado y del alumnado en lo relativo a la educación sexual y cómo esta estrecha vinculación se materializa en la creación de materiales actualizados que tienen en cuenta sus necesidades.

- Fomentar el desarrollo de perspectivas no adultocéntricas en el tratamiento de la juventud como sector poblacional y de aquellos espacios de socialización que les son propios, en concreto, las redes sociales.

\section{Metodología}

La investigación planteada en este texto parte de un estudio de caso que pretende abordar un fenómeno contemporáneo dentro de un contexto local. Si bien los estudios de caso son particularistas, descriptivos y heurísticos y se basan en el razonamiento inductivo al manejar múltiples fuentes de datos (Pérez, 1994), también permiten establecer un diálogo entre los discursos culturales que operan en torno a la significación cultural que adquiere la educación sexual y las respuestas ofrecidas por el Conseyu de la Mocedá, sin perder de vista que su actividad se realiza desde la educación no formal. A través de una metodología esencialmente cualitativa se persigue adentrarse en el planteamiento de un problema de carácter dinámico, interactivo y en evolución, lo que, a su vez, permite analizar comprehensiva, profunda y exhaustivamente situaciones y campos particulares y posibilita probar hipótesis y desarrollar teorías (Martínez, 2006; Hernández y Mendoza, 2008).

En la aproximación teórica realizada se ha apostado por una revisión y recopilación sistemática de la literatura científica y documental sobre la temática investigada (Hernández et al., 2014): publicaciones académicas, datos estadísticos institucionales, descripciones de programas o legislación relacionada con el fenómeno estudiado, además de registros, archivos y bases de datos facilitados por Conseyu de la Mocedá del Principáu d'Asturies. También se ha recogido información aplicando diferentes técnicas como la observación directa no estructurada, la entrevista personal no estructurada (McMillan y Schumacher, 2005; Martínez, 2006) y la observación participante (Kawulich, 2005).

Los materiales que serán el foco de este texto pueden enmarcarse en lo que ha sido definido como educación sexual mediática y concretamente dentro de un esfuerzo del Conseyu por hacer a la juventud participe de un proceso de alfabetización mediática, es decir:

no se trata en exclusiva de saber gestionar técnicamente las redes sociales ni las aplicaciones de mensajería instantánea de moda, sino en reflexionar cómo esos medios tecnológicos y de comunicación, así como los mensajes que se desprenden de su uso y presencia van a ser relevantes para la vida diaria, van a acompañarnos en el crecimiento personal, y van a ser objeto de aprendizaje, conocimiento y crítica. (Calvo, 2015: 203)

El análisis de los datos recogidos se centra en la comprensión de las dinámicas socioculturales que estructuran la producción de los distintos materiales educativos elaborados por el organismo. Esta compilación será comprendida como una genealogía sobre las propias necesidades educativas diagnosticadas por profesionales implicados en su elaboración, pero también como un testimonio de la evolución de la propia disciplina de la educación sexual. Desde un papel participativo, los autores y la autora de este artículo abordan este análisis no desde una perspectiva externa y aséptica, sino desde su propia colaboración con el Conseyu de la Mocedá. Esto es considerado como una fortaleza en la rigurosidad planteada, ya que esta investigación-acción permite comprender y resolver problemáticas específicas y evidenciar las dinámicas internas de trabajo.

\section{Resultados}

Los materiales analizados en este artículo han sido planteados como guías dirigidas a diferentes profesionales en las que se recogen las herramientas teóricas necesarias junto con dinámicas propuestas para su trabajo con jóvenes. Todas ellas incluyen indicaciones y recursos con las que generar un espacio de debate e intercambio de opiniones entre el alumnado, pero a su vez, se deja espacio para la adaptación a diferentes niveles y particularidades de cada grupo. En este sentido, el papel activo del profesorado y demás personas formadoras es fundamental en la detección de las necesidades y conflictos surgidos para poder establecer las líneas de trabajo sirviéndose, para ello, de los materiales aquí descritos.

Materiales educativos elaborados por el Conseyu de la Mocedá (20142020)

A partir del año 2014 el Conseyu de la Mocedá del Principau d'Asturies comienza a elaborar una serie de materiales didácticos que tienen como objetivo facilitar recursos para abordar la educación sexual desde una mirada situada en la realidad tecnológica y digital que viven las personas jóvenes. Las Tecnologías de la Relación, Información y Comunicación (TRICs) forman parte de nuestras realidades cotidianas, constituyéndose como uno de los fenómenos más característicos del escenario social contemporáneo. Internet ha cambiado nuestra forma de ser, vivir y expresarnos, definiendo lugares no territoriales de comunicación y encuentro. Lo digital se presenta como un espacio en el que habitar $\mathrm{y}$, por lo tanto, como un lugar en el que se configuran nuestras vivencias, identidades y relaciones, y desde el que se expresa y se conoce el mundo que nos rodea, especialmente para las personas más jóvenes que se han convertido en las primeras generaciones nativas digitales.

Según el estudio "Las TIC y su influencia en la socialización de los adolescentes" (Ballesteros y Picazo, 2018), basado en el análisis de uso de las personas adolescentes de entre 14 y 16 años en España, cerca del $90 \%$ disponen de entre dos y cinco dispositivos personales, destacando entre ellos, en primer lugar, el smartphone $(89,9 \%)$, seguido por el ordenador portátil $(76 \%)$ y la tablet (69\%). Además, una gran parte $(76,7 \%)$ afirma tener estos dispositivos desde hace al menos dos años, entre los 11-12 años, y utilizarlos de manera habitual y cotidiana $(75,6 \%)$. Respecto a las redes sociales, se constituyen como un canal básico de conexión con el entorno social: casi la totalidad de los y las adolescentes encuestados/as (92\%) afirman tener un perfil propio, sin quedar apenas jóvenes que no dispongan de uno $(5,8 \%)$. Entre sus preferencias destacan plataformas como Instagram, la principal red social para publicar contenidos propios y ser vistos/as, Youtube para consumir contenidos digitales, WhatsApp como herramienta para el contacto cotidiano con amistades y familiares, así como Twitter y TikTok, siendo esta última la que más está calando entre los menores de 16 años. Dentro de este contexto, las app para ligar se han convertido también en un lugar en el que establecer vínculos, interactuar y comunicarse (Fernández y Gómez, 2019) y la pornografía gratuita en Internet en una de las principales fuentes de información sexual, ofreciendo un imaginario cargado 
de mitos y estereotipos sobre la sexualidad humana (Ballester et al., 2014; Ballester y Orte, 2019; Sanjuán, 2020; Rodriguez, 2020).

Todos estos datos, confirman finalmente la intensiva y extensa digitalización de los y las jóvenes y la temprana edad a la que tienen acceso a las TRICs. Además, hacen evidente que el mundo digital es algo fundamental en los procesos de socialización de la adolescencia, así como de la construcción de sus identidades, sus imaginarios eróticos y de los procesos de participación e inclusión social. Como explican Megías y Rodríguez (2014: 7):

La adopción, omnipresencia y ubicuidad de estos dispositivos no es una mera cuestión cuantitativa. Su amplia difusión, personalización y la posibilidad de conexión permanente que crean, contribuyen a reconfigurar numerosos aspectos de la vida cotidiana y así como de los procesos de subjetivación y socialización contemporáneos.

Los usos y mediaciones tecnológicas están transformando nuestras vivencias y experiencias personales y colectivas, así como las formas en las que se configuran nuestras identidades, nuestras relaciones y vinculaciones afectivas, nuestros deseos, eróticas, experiencias y procesos de seducción. Por esta razón, desde el Conseyu de la Mocedá del Principau d'Asturies se comienzan a generar una serie de materiales didácticos que facilitan abordar los entornos digitales y mediáticos en clave sexológica y de género.

\section{Propuestas educativas sobre el uso y gestión de RRSS}

"Nuevos modelos de comunicación en parejas jóvenes: propuestas para la intervención en entornos socioeducativos" (2017)

Como se ha comentado, una de las dificultades más habituales en el trabajo con las TRICs es la velocidad con la que estas se han integrado en nuestras vidas, especialmente en las de las personas jóvenes, y continúan cambiando y transformando la manera en la que se relacionan en el espacio digital. Ante esta situación, Calvo (2017), de mano del Conseyu de la Mocedá, decidió elaborar una propuesta de intervención socioeducativa a partir de un estudio de campo en el que se analizó la manera en la que las personas jóvenes interactúan en las redes sociales para así "adentrarnos en el conocimiento de las identidades digitales y sus interacciones a partir de las relaciones interpersonales, afectivas y emocionales que se establecen mediante las TIC" (Calvo, 2017: 11).

Esta propuesta continúa con la línea de trabajo del Conseyu de la Mocedá de elaborar materiales que partan de la experiencia propia de las personas jóvenes, de sus necesidades y experiencias, así como de los obstáculos que encuentran en una sociedad que menosprecia sus fórmulas relacionales dentro y fuera del espacio virtual. Así mismo, plantea una comprensión de las redes sociales como espacios de intercambio "de acercamiento, encuentro, disfrute y vivencia común” (Calvo, 2017: 23), poniendo el centro no ya en la demonización de estos espacios, sino más bien en la necesidad de una educación sexual mediática que aborde el desarrollo de la responsabilidad individual y colectiva en el uso de estas plataformas.

Este material se divide en tres partes. Una introducción junto con la explicación metodológica oportuna en la que se detalla la colaboración de centros educativos asturianos, así como la creación de perfiles en Instagram y Twitter para conocer de primera mano sus experiencias. Una segunda parte en la que se mencionan las principales conclusiones obtenidas tras el análisis de los datos. Y en último lugar se incluyen las diferentes propuestas para trabajar las identidades digitales en entornos educativos formales y no formales en el que se incorporan preguntas generadoras, enlaces a materiales complementarios y consejos para las personas que realicen la mediación.
“Nuevos Espacios de Seducción” (2019)

Los esfuerzos por abarcar aquellos espacios de relación interpersonal específicos de la población joven también se han dirigido al análisis de las aplicaciones de contactos. La guía "Nuevos espacios de seducción" (Fernández y Gómez, 2019) se convierte así en una herramienta reflexiva que aborda desde una perspectiva comprensiva el mundo de las apps de citas entre las personas jóvenes, ya que tienen un gran impacto entre este colectivo -sobre todo en menores de 35 años-, tal y como reflejan las estadísticas y estudios académicos que se muestran en ella. A través de estas apps, las personas jóvenes desean conectar y establecer todo tipo de vínculos con otras personas (caer bien, hacer nuevas amistades, gustar, resultar deseables, enamorar...), al mismo tiempo que buscan cubrir la necesidad de reciprocidad que forma parte de cualquier intercambio comunicativo. Esto es planteado a través del concepto de "seducción", reflejando esas ganas de conexión y de mutualidad presentes en estos intercambios virtuales (Fernández y Gómez, 2019).

Esta guía está dirigida al personal docente y educador, formadores/as, profesionales, instituciones y personas adultas. El documento consta de una parte teórica y divulgativa, una unidad didáctica dividida en cuatro actividades destinadas a trabajar con el alumnado los contenidos que se abordan a lo largo del documento para profundizar colectivamente en el conocimiento de las redes, las apps y el uso que hacemos de ellas haciendo hincapié en la responsabilidad, el buen trato y el carácter diverso de las experiencias que se dan en ellas. Así mismo, incorpora un glosario para aclarar términos que pueden resultar confusos y un apartado de información sobre los servicios de asesoramiento sexual gratuito para jóvenes en Asturias. Como se refleja en las páginas introductorias del material:

El fin último de esta guía es estimular la reflexión individual y colectiva a través de la educación sexual mediática crítica y con perspectiva de género, basada en la diversidad e incorporando estos nuevos modelos de interacción para "suscitar elementos de cultivo" (Fernández y Gómez, 2019: 10).

Es en este contexto complejo donde las apps móviles y las redes sociales han llegado para quedarse, especialmente entre las personas jóvenes, como espacios que ofrecen nuevas formas de construcción de identidades, nuevos lenguajes y nuevas formas de comunicación, de interacción y, sobre todo, de relación (Medicus Mundi Sur, 2018); pero también espacios de negociación de las subjetividades, la intimidad y la privacidad (Enguix y Gómez-Narváez, 2017).

Propuestas educativas sobre eróticas mediáticas

“Sexting Positivo: propuesta didáctica para $2^{\circ}$ ciclo de la ESO" (2015)

Tanto el proyecto "aSEXora WhatsApp" como la guía "Identidades digitales" permitieron constatar el incremento del impacto de las redes sociales en el establecimiento de redes comunicativas entre las personas jóvenes ${ }^{1}$. Los móviles no solo estaban mediando las relaciones, sino que, además, también comenzaban a dar lugar a nuevas maneras de establecer vínculos también en términos eróticos. Si bien desde un inicio los esfuerzos del Conseyu de la Mocedá se habían orientado a la promoción de una educación

El servicio "aSEXora WhatsApp" fue creado en 2012 con la intención principal de ofrecer un punto de asesoramiento a la población joven de Asturias, utilizando la aplicación de mensajería instantánea para establecer un canal de comunicación efectivo con la juventud. Teniendo como base este servicio, en el año 2014 se elaboró la guía "Identidades digitales" en la que su autora, Soraya Calvo, preparó una unidad didáctica de apoyo al programa de educación sexual "Ni ogros ni princesas". 
sexual que estimulara la responsabilidad y el buen trato dentro y fuera de lo virtual, es a través de la guía editada en papel "Sexting positivo" (Calvo, 2015) donde estos esfuerzos se concretan en una propuesta didáctica para el segundo ciclo de la ESO.

Este material propone abordar el sexting, es decir, el intercambio consensuado de material erótico, desde una visión no adultocéntrica, abandonando posturas que infravaloren la autonomía y la libertad de las personas jóvenes, todo ello evitando hacer hincapié en el miedo como un recurso pedagógico. Es precisamente por esta razón, por la que se dedican varios apartados a concretar aquello que es el sexting, distinguiéndolo de prácticas delictivas como el sexpreading, y volviendo a incidir en el carácter "consensuado", "consciente" y "transparente" de esta práctica erótica:

“[n]o es algo 'malo', ni un problema: es una práctica erótica consensuada entre dos o más personas que tienen una relación personal de algún tipo, con una finalidad positiva y de disfrute, y en la que todas las personas implicadas tienen la capacidad de tomar decisiones" (Calvo, 2015: 6).

También se ofrecen algunos consejos prácticos para poder optimizar nuestra seguridad a la hora de llevar a cabo esta práctica, por ejemplo, eludiendo enseñar partes del cuerpo fácilmente reconocibles por tatuajes o piercings; evitar enseñar la cara; y prestar atención a las plataformas que se usan, pudiendo decantarnos por aquellas que nos ofrecen herramientas para borrar nuestras imágenes una vez enviadas. Así mismo, para concluir este material se ofrecen al profesorado cuatro dinámicas con las que ahondar en la importancia de desarrollar una perspectiva crítica, empática y de responsabilidad afectiva. Se propone el trabajo con noticias y situaciones reales, con vídeos que permiten aclarar conceptos junto con distintas estrategias pedagógicas para potenciar el establecimiento de una comunicación más efectiva y mejor gestión de los conflictos.

"Construcción del imaginario sexual en las personas jóvenes: la pornografía como escuela" (2020)

El material más reciente editado por el Conseyu de la Mocedá hasta la fecha va a poner de manifiesto una realidad notoria en lo referente a la educación sexual de las personas jóvenes: la trascendencia de la pornografía en la configuración del imaginario sexual juvenil debido a la ausencia de una educación sexual reglada y obligatoria en las diferentes etapas educativas.

Según los últimos estudios realizados en España sobre el consumo de pornografía durante la adolescencia (Ballester et al., 2014; Ballester y Orte, 2019; Sanjuán, 2020), las edades de inicio son cada vez más reducidas, en torno a los 12 años, y el consumo es frecuente. De esta forma, hablamos de la generación $\mathrm{Z}$ como una generación pornonativa: jóvenes "cuyas prácticas, gustos y preferencias sexuales tienen que ver más con las experiencias observadas en el porno que con las experiencias vividas" (Rodríguez, 2020: 14). La pornografía se ha convertido así en una de las principales fuentes de información sobre sexualidad, siendo uno de los principales motivos de su consumo el aprendizaje sexual: es muy significativo que casi la mitad de los y las adolescentes (el $48 \%$ ) la validan como fuente de aprendizaje y que para el $30 \%$ es la única fuente de información (Sanjúan, 2020: 65).

En este contexto, la guía "Construcción del imaginario sexual en las personas jóvenes: la pornografía como escuela" (Rodríguez, 2020) surge de la necesidad de abordar los mensajes distorsionados sobre la sexualidad que ofrece la pornografía desde la perspectiva de la educación sexual. Frente a los discursos prohibicionistas, esta guía propone facilitar una educación sexual mediática que les ayude a gestionar los contenidos pornográficos desde una mirada crítica ante la información que reciben y que comparten.

Este material ofrece herramientas y recursos pedagógicos para que el profesorado, las familias y otros agentes sociales puedan comprender cómo están influyendo los contenidos pornográficos en el imaginario sexual de las personas jóvenes y puedan acom- pañar este visionado facilitando una reflexión crítica. Para ello cuenta con una parte más divulgativa, en la que se revisan cómo presenta la pornografía los cuerpos, las eróticas, las prácticas de riesgo, los roles de género, las relaciones de poder, los modelos relacionales o la violencia sexual, y en la que se profundiza en la importancia de transmitir el mensaje de que el porno es ficción, de que las situaciones que muestra no son reales y que, por tanto, tratar de reproducirlas no tiene ningún sentido. Por otro lado, cuenta con una serie de actividades didácticas diseñadas para reflexionar críticamente con personas jóvenes, a partir de 16 años, acerca de los diversos mitos y falsas creencias relacionadas con la sexualidad que propone la pornografía, fomentando un modelo sexual basado en la igualdad, la diversidad y los buenos tratos.

\section{Discusión y conclusiones}

Vinculados con el primer objetivo específico, los resultados reflejan que los materiales seleccionados y analizados son recursos útiles y valiosos para abordar la educación sexual en contextos no formales, ya que están diseñados como un recurso educativo dirigido a entidades y profesionales que, sin disponer de una formación o una trayectoria especializadas en cuestiones de sexualidad, sean capaces de abordar estos saberes de forma rigurosa, científica, sistemática y pedagógica como complemento formativo de los currículos académicos; es decir, garantizar que cada profesional y cada organismo sepa jugar su papel y cumplir su función sin convertirse en meros ejecutores o reproductores de programas o materiales, sino en profesionales críticos y reflexivos (Barragán, 1995) que garanticen el pleno desarrollo y disfrute de los Derechos Sexuales y Reproductivos a los colectivos y grupos con los que intervienen.

Vinculado con el segundo objetivo específico, se evidencia la importancia de elaborar, diseñar y desarrollar tanto publicaciones como intervenciones educativas actualizadas que recojan las necesidades del alumnado y, a su vez, contribuyan a la formación continua de profesionales y personas mediadoras que se ocupen de abordar la educación sexual mediática desde espacios no formales como el Conseyu de la Mocedá del Principáu d'Asturies. En este sentido, es imprescindible plantear acciones destinadas a mejorar la capacitación profesional y el apoyo a la formación inicial y continua del colectivo docente y del resto de especialistas que trabajan en ámbitos relacionados con la sexualidad (OMS, 2010; Hurtado et al., 2012; UNESCO, 2016).

Vinculado con el tercer objetivo específico, consideramos necesario desarrollar perspectivas no adultocéntricas en el abordaje de la educación sexual desde planteamientos que no solo aborden los peligros, sino una perspectiva positiva de la sexualidad, así como que se preste atención a los elementos mediáticos que consumen las personas jóvenes y que puedan adaptarse mejor al desarrollo, las vivencias y las problemáticas derivadas de la convivencia cotidiana con las TRICs.

Las limitaciones de este estudio radican en la ausencia de una estrategia de evaluación estandarizada de estos materiales y en las particularidades propias del estudio de caso, como los posibles sesgos derivados de la vinculación del equipo investigador con el Conseyu de la Mocedá del Principáu d'Asturies, así como las dificultades y las pocas bases que este método proporciona para generalizar los resultados y las conclusiones (Martínez, 2006).

\section{Agradecimientos}

Esta investigación no ha sido financiada por ninguna entidad.

\section{Contribución individual de los autores y autoras al manuscrito}

La elaboración del manuscrito se ha repartido conjuntamente entre las personas encargadas de su realización. Aun así, pueden destacarse varias áreas en las que cada una de las personas integrantes ha prestado más atención. En primer lugar, DFF se 
ha encargado del marco legislativo y sexológico, junto con la descripción de materiales analizados. En segundo lugar, MRS ha incidido en el marco sexológico así como en lo relativo a las especificidades relacionadas con el Conseyu de la Mocedá y el análisis de los materiales propuestos. Por último, la aportación de IGB se ha centrado en el análisis de materiales específicos de redes sociales así como en la revisión completa.

\section{Referencias}

Ballester, L. y Orte, C. (2019). Nueva pornografía y cambios en las relaciones interpersonales. Octaedro.

Ballester, L., Orte, C. y Pozo, R. (2014). Estudio de la nueva pornografía y relación sexual en jóvenes. Anduli. Revista Andaluza de Ciencias Sociales, 13, 165-178. http://dx.doi.org/10.12795/ anduli.2014.i13.1

Ballesteros, J. C. y Picazo, L. (2018). Las TIC y su influencia en la socialización de adolescentes. FAD.

Barragán, F. (1995). Curriculum, poder y saber: Un análisis crítico de la educación sexual. Anuario de sexología, 1, 83-90.

Bejarano, M. y García, B. (2016). La educación afectivo-sexual en España. Análisis de las leyes educativas en el periodo 19902016. Opción, 32(13), 756-789.

Bejarano, M. y Jiménez, A. (2016). La educación afectivo-sexual en el sistema educativo español: análisis normativo y posibilidades de investigación. Revista Ibero-Americana de Estudos em Educação, 10(6), 1507-1522. https://doi.org/10.21723/riaee. v10i6.8334

Bejarano, M. y Mateos, A. (2016). Reflexiones y propuestas para mejorar la educación afectivo-sexual en España. En P. Regina Costa Ribeiro, E. Pinheiro de Queiroz Silva y F. Teixeira (Eds.), Atravessamentos de genero, corpos e sexualidades: Linguagens, Apelos, desejos, posibilidades e desafíos (pp.149-172). Universidad Federal Do Rio Grande-FURG.

Calvo, S. (2015). Educación sexual mediática. Incorporando la alfabetización mediática crítica en un programa de educación sexual para educación secundaria obligatoria. Redes.com: revista de estudios para el desarrollo social de la comunicación, 12, 194221. https://doi.org/10.15213/redes.n12.p194

Calvo, S. (2017). Nuevos modelos de comunicación en parejas jóvenes: Propuesta para la intervención en entornos socioeducativos. Conseyu de la Mocedá del Principáu d'Asturies.

Calvo, S. (2021). Educación sexual con enfoque de género en el currículo de la educación obligatoria en España: avances y situación actual. Educatio Siglo XXI, 39(1), 281-304. http://dx.doi. org/10.6018/educatio.46928

Consejo de la Juventud de España (s.f.). ¿Qué es el CJE?. Disponible en https://bit.ly/3k8vLrx

Conseyu de la Mocedá del Principáu d'Asturies (s.f.). CMPA. Disponible en https://bit.ly/2UHHiDv

De la Cruz, C. (2003). Educación de las Sexualidades: los puntos de partida de la educación sexual. Revista española de sexología, 119, 1-122.

Enguix, B. y Gómez, E. (2017). Masculine Bodies, Selfies, and the (Re)configurations of Intimacy. Men and Masculinities, 21(1), 112-130. https://doi.org/10.1177/1097184X17696168

Fernández, D. (2018). Educación sexual en el Grado en Pedagogía. Diagnóstico educativo y propuestas para la mejora. Universidad de Oviedo. [Trabajo Fin de Máster]

Fernández, D. y Calvo, S. (2020). Inclusión de la comunidad educativa y los agentes sociales en los procesos de innovación de planes docentes universitarios sobre educación sexual. En J.J. Gázquez Linares, M. Molero Jurado, A. Martos Martínez, et al. (Coord.), Investigación en el ámbito escolar. Nuevas realidades en un acercamiento multidimensional a las variables psicológicas y educativas (pp. 717-728). Dykinson.
Fernández, D. y Gómez, I. (2019). Nuevos espacios de seducción. Conseyu de la Mocedá del Principáu d'Asturies.

Garzón, A. (2016). La educación sexual, una asignatura pendiente en España. Revista Biografía Escritos sobre la biología y su enseñanza, 9(16), 195-203. https://doi.org/10.17227/20271034. vol.9num.16bio-grafia195.203

Hernández, R., Fernández, C. y Baptista, P. (2014). Metodología de la investigación. Editorial Mc Graw Hill.

Hernández, R. y Mendoza, C. (2008). El matrimonio cuantitativo-cualitativo: el paradigma mixto. En J.L. Álvarez Gayou (Presidente). 6ํㅡㅁ Congreso de Investigación en Sexología. Congreso efectuado por el Instituto Mexicano de Sexología, AC y la Universidad Juárez Autónoma de Tabasco, Villahermosa, Tabasco, México.

Hurtado, F., Pérez, M., Rubio, E., Coates, R., Coleman, E., Corona, E., E. Mazín y Horno, P. (2012). Educación para la sexualidad con bases científicas. Documento de consenso de Madrid. Recomendaciones de un grupo internacional de expertos. En F. Hurtado Murillo y M. Pérez Conchillo (Coord.), Encuentro Mundial llevado a cabo por la Asociación Española de Especialistas en Sexología-AEES, la Academia Española de Sexología y Medicina Sexual-AESMES, la Asociación Mundial para la Salud Sexual-WAS.

Kawulich, B. (2005) La observación participante como método de recolección de datos. Forum Qualitative Social Research, 6 (2), 1-32.

Lameiras, M., Carrera, M. y Rodríguez, Y. (2016). Caso abierto: la educación sexual en España, una asignatura pendiente. En V. Gavidia (Coord.), Los ocho ámbitos de la Educación para la Salud en la escuela (pp. 197-210). Tirant lo Blanch.

Ley 1/1986 de 1986, del Consejo de la Juventud del Principado de Asturias, 31 de marzo de 1986. BOPA núm. 81.

Ley Orgánica 8/2013 del 2003, para la Mejora de la Calidad Educativa, 10 de diciembre del 2013. BOE núm. 295.

Martínez, J., Baz, B., Molina, I., Ortega, E., González, E., Fuertes, A. y Martín, J. (2011). Educación sexual y formación del profesorado en España: diferencias por sexo, edad, etapa educativa y comunidad autónoma. Magister: Revista miscelánea de investigación, 24, 37-47.

Martínez, P. C. (2006). El método de estudio de caso. Estrategia metodológica de la investigación científica. Revista científica Pensamiento y Gestión, (20), 165-193.

McMillan, J. y Schumacher, S. (2005). Investigación Educativa: Una Introducción conceptual. Pearson Educación.

Medicus Mundi Sur (2018). Adolescencias y cuerpos. Transformando realidades. TRICS. Andalucía. Disponible en https://bit.ly/2SQK0Wj

Megías, I. y Rodríguez, E. (2014). Jóvenes y comunicación. La impronta de lo virtual. Centro Reina Sofía sobre Adolescencia y Juventud y FAD.

Organización Mundial de la Salud, Oficina Regional de la OMS para Europa y BZgA (2010). Normas para la educación sexual en Europa: Marco para los responsables de la formulación de políticas, las autoridades y los especialistas en educación y salud. Centro Federal de Educación para la Salud.

Pérez, G. (1994). Investigación cualitativa. Retos, interrogantes y métodos. La Muralla.

Rodríguez, M. (2020). Construcción del imaginario sexual en las personas jóvenes. La pornografía como escuela. Conseyu de la Mocedá del Principau d'Asturies.

Sanjuán, C. (2020). (Des)información sexual: pornografía y adolescencia. Save the Children

Soares, M. y De Toledo, M. (2012). Dialogando con estudios acerca de las vivencias afectivo-sexuales de las parejas de transexuales. Liberabit, 18(2), 125-129.

UNESCO (2016). Proyecto de estrategia de la UNESCO sobre la educación para la salud y el bienestar: contribución a los objetivos de desarrollo sostenible. UNESCO. 